\title{
Ernst Kern: Personzentrierte Körperpsychotherapie
}

\author{
Ernst Reinhardt Verlag, 2. Aufl. 2015, München, \\ 186 Seiten, 24,90€ (D)
}

Ernst Kern legt ein überzeugendes, theoretisch gut fundiertes Praxisbuch zur Körperpsychotherapie vor, dessen Reichhaltigkeit sich merklich aus 20 Jahren klinischer Erfahrung speist. Des Autors Referenzsystem ist seine alltägliche Arbeit im Rahmen stationärer Psychiatrie, basierend auf einem verhaltenstherapeutischen und gesprächspsychotherapeutischen Hintergrund, unterfüttert von immer wieder deutlich herausgearbeiteten Bezügen zu Gendlins Focusing-Modell.

Übersichtlich gliedert sich das Buch in sieben Kapitel, die nicht zwangsläufig nacheinander gelesen werden müssen. Im ersten Kapitel werden die phänomenologischen Grundlagen des vorgestellten Denkansatzes erläutert, wobei Erfahrung und Erleben als zentrale Ausgangspunkte des von Kern „personzentriert“ genannten Körperpsychotherapie-Ansatzes erklärt werden. Die ursprünglich humanistische Richtung der „Gesprächspsychotherapie“ wurde bekanntlich terminologisch zur „Personzentrierten Psychotherapie“, und hier liegt denn auch die Wurzel für den Titel des vorliegenden Buches. Es handelt sich um eine immer wieder auf die humanistische Tradition bezogene Zusammenschau verschiedener Theorien aus unterschiedlichen Disziplinen, die in einen Bezug zu ausgewählten Praxisansätzen aus der Körperpsychotherapie gestellt werden.

In Kapitel zwei werden „erweiternde Perspektiven“ eröffnet. Der Autor orientiert sich am „state of the art" der aktuellen Psychotherapie-Diskussion und nimmt eine erfrischend klare, übrigens gleichzeitig integrierende, aber auch differenziert abgrenzende Einordnung seines körperpsychotherapeutischen Arbeitsansatzes vor: So greift er Forschungsrichtungen auf und stellt deren Bedeutung für eine elaborierte Anwendung körper-und leibfundierter Interventionen in der klinischen Psychotherapie einmal mehr klar. Ergebnisse aus der
Säuglingsforschung und der psychoanalytisch fundierten Entwicklungstheorie nach Daniel Stern werden, auch unter Berücksichtigung des zen-

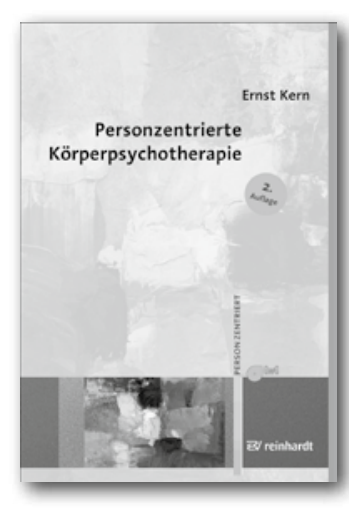
tralen Konzepts des „now-moments“ für das Patienten-Erleben, zusammengefasst.

Der neurowissenschaftliche Diskurs inkl. der Embodiment-Forschung wird ausschnittartig mit besonderem Bezug auf Damasios Modell der „somatischen Marker" gut nachvollziehbar entfaltet. Auch die Bedeutung von Bowlbys Bindungstheorie (welche die Diskussion in allen psychotherapeutischen Verfahren hinsichtlich der Wichtigkeit einer stimmigen nonverbalen Beziehung zwischen Baby und früher Bezugsorganisation bereichert hat) wird dem Leser klar. Der Ansatz Lesley Greenbergs (eine aktuell einflussreiche Emotionstheorie) wird ebenso zutreffend als ganzheitlicher Ansatz mit Fokussierung auf physiologische, affektive wie auch kognitive Erfahrungen dargestellt, wie George Downings mehr als 20 Jahre altes tiefenpsychologisches Konzept, in dem schon damals menschliche Emotionen aus dem subjektiven Körpererleben hergeleitet wurden. Das Modell des affektiven Zyklus nach Geuter und Schrauth erweitert der Autor kreativ um den „Pol des exzessiven Gefühlsausdrucks", welcher bei Borderline- und Traumapatienten häufig ausschlägt. Diese selbstbewusste Weiterentwicklung ist stimmig und hat mir besonders gut gefallen.

Schließlich wird der „Achtsamkeitsansatz“ aus der sogenannten „dritten Welle der Verhaltenstherapie“ in den Kontext der Körperpsychotherapie zurückgeführt; denn unter "Achtsamkeit“ ist ein übergeordnetes Prinzip der „Weisheit des Körpers" zu verstehen, das in den letzten Jahren auch 
in der („modernen“) Verhaltenstherapie und in der Tiefenpsychologie rezipiert wird.

Den fachlich interessierten Leser erfreut nach diesen ersten 80 Seiten des Buches besonders, dass nach jedem erläuternden Unterpunkt „Schlussfolgerungen für die personzentrierte Körperpsychotherapie“ gezogen werden. Deutlich markierte Zusammenfassungen und Überblicksabsätze erleichtern dadurch auch dem „schnellen Leser“ den Blick auf das Wesentliche.

In Kapitel drei widmet sich der Autor dann einer „den Körper selbstverständlich einbeziehende(n) ganzheitliche(n) Sichtweise“. Erfrischend deutlich wird einmal mehr, dass die zurzeit als besonders modern daher kommenden manualisierten Ansätze der o.a. sogenannten dritten Welle der Verhaltenstherapie (CBASP, DBT, ACT) als das benannt werden, was sie sind: gut strukturierte Zusammenstellungen von hilfreichen Elementen, die mit Bezug auf Erleben und Handeln den Patienten helfen können, sich selbst besser (ganzheitlich) wahrzunehmen und zu strukturieren.

Nach einer ausführlichen Auseinandersetzung mit den Therapeutenbedingungen (körperbasierte Empathie, „energetische“ Wahrnehmung, Erhebung einer körperorientierten Anamnese, bedingungsfreie Anerkennung und Achtsamkeit, körperbasierte Kongruenz und Präsenz) wird nun unter Verwendung praktischer Beispiele eine starke Parallele zum verbalen personzentrierten Vorgehen (mit sehr deutlichen Bezügen zu Rogers) deutlich. Folglich werden im nächsten Unterkapitel die Klientenbedingungen dargelegt, mit hervorgehobenem Bezug auf Gendlins Focusing-Modell und damit dem subjektiven Erleben als Ausgangspunkt allen therapeutischen Handelns. Praxisrelevante Arbeitsblätter ermutigen den therapeutisch ausgebildeten Leser, die hier zusammengestellten Techniken mit eigenen Patienten zu erproben. Kapitel 4 vertieft diesen praktischen Zugang: Weitere Fallbeispiele und Arbeitsblätter zu Wahrnehmung, Atmung, Haltung, Bewegung und Körperkontakt laden den Therapeut / Leser zur Anwendung ein - übrigens mit der Freiheit, dabei das eigene handlungsleitende Paradigma zu nutzen.

Kapitel 5 beschäftigt sich mit einem „Alphabet der Leiblichkeit“. Hierbei wird auf das Körperselbstbild wie auch auf das Polaritätsprinzip aus der Tanztherapie und auf Labans Kategorien der Bewegungsantriebe zurückgegriffen. In diesem Kapitel hätte ich mir die Zitierung von vermutlich genutzten weiteren Quellen gewünscht, entsteht doch an dieser Stelle der sonst im gesamten Buch vermiedene Eindruck, aus bereits veröffentlichten, systematisierten Übungskatalogen von Kollegen ein wenig gewildert zu haben - ohne aktive Bezugnahme auf diese Quellen. Das ist schade und wäre angesichts der ansonsten souveränen Wissensfülle des Autors nicht nötig gewesen.

Kapitel 6 bezieht sich dann, wieder exakt zitierend, auf das Phasenentwicklungsmodell von Röhricht und stellt körperorientierte Angebote zur Regulation von Spannung und Erregung, zum Grounding, zur Erfahrung von Raum und Rhythmus u.a. dar. Hier wird auch sehr schön erneut eine praktische Verbindung zur Stern'schen Theorie des Selbst-Erlebens hergestellt. Und es werden die wichtigen Themen „Berührung“ und „narrative Einordnung von Erlebtem“ behandelt. In Kapitel 7 wird sodann eine störungsspezifische Perspektive eingenommen. Erscheinungsformen, körperliches Erleben sowie mögliche körperpsychotherapeutische Interventionen werden bei den wichtigsten klinischen Störungsbildern umrissen: Depression, Angst, Sucht, BorderlinePersönlichkeitsstörung und Traumafolgestörung.

Insgesamt handelt es sich um ein empfehlenswertes, klar aufgebautes und anregendes Fachbuch, das mit einer Fülle von Praxiswissen aufwartet und einen fokussierten Blick auf eine ganzheitlichklinische Arbeit richtet, in der menschliches Erleben im Vordergrund steht, was natürlich die Leiblichkeit immer mit einschließt.

Dr. Sabine Trautmann-Voigt

DOI 10.2378/ ktb2016.art13d 